\title{
Competências em promoção da saúde: conformações e recursos mobilizados na residência multiprofissional
}

\section{Competences in health promotion: conformations and resources mobilized in the multiprofissional} residency

\section{Competencias en promoción de la salud: conformaciones y recursos movilizados en la residencia} multiprofesional

Lucas Dias Soares Machado ${ }^{1,2}$ (c) Samyra Paula Lustoza Xavier ${ }^{1,2}$ []

Paloma Loiola Leite ${ }^{1}$ (1)

Maria Rosilene Cândido Moreira ${ }^{3}$ (C)

Maria Rocineide Ferreira Silva ${ }^{2}$ (D)

Maria de Fátima Antero Sousa Machado 4 (1)

1. Universidade Regional do Cariri,

Departamento de Enfermagem. Iguatu, CE, Brasil.

2. Universidade Estadual do Ceará, Programa de Pós-Graduação em Cuidados Clínicos em Enfermagem e Saúde. Fortaleza, CE, Brasil.

3. Universidade Federal do Cariri, Programa de Pós-Graduação em Desenvolvimento Regional Sustentável. Juazeiro do Norte, CE, Brasil.

4. Fundação Oswaldo Cruz, Programas de PósGraduação em Saúde da Família. Fortaleza, $\mathrm{CE}$, Brasil.

\section{Resumo}

Objetivo: reconhecer a conformação de competências em promoção da saúde na residência multiprofissional em saúde. Método: estudo qualitativo, realizado junto aos programas de residência multiprofissional do estado do Ceará e orientado pelo referencial do Competences in Health Promotion Project (CompHP). Participaram 13 profissionais do colegiado docente de cinco programas de residência com atuação prioritária na atenção básica. Os dados foram coletados por meio de entrevistas semiestruturadas e interpretados por meio de dendrograma organizado no ALCESTE. Resultados: duas classes foram originadas relacionando as concepções do colegiado docente da residência sobre competências, competência em promoção da saúde e seu desenvolvimento a partir do processo formativo da residência multiprofissional. O desenvolvimento de competências em promoção da saúde na residência multiprofissional envolve a articulação de atributos psicomotores, cognitivos e afetivos. Para tanto, valorizam-se as práticas coletivas e multiprofissionais, utilizando estratégias de comunicação, educação popular e identificação de parcerias, entre outros. Considerações finais e implicações para a prática: fomentar o desenvolvimento de competências em promoção da saúde contribui com a superação de modelos tradicionais de ensino e práticas de saúde, entretanto, aspectos políticos, institucionais e sociais destacam a necessidade de cautela no desenvolvimento de estudos pautados na formação de equipes multiprofissionais, representando limitações do estudo.

Palavras-chave: Capacitação de Recursos Humanos em Saúde; Competência Profissional; Educação Baseada em Competências Internato não Médico; Promoção da Saúde.

\section{Abstract}

Objective: to recognize the conformation of competences in health promotion in the multidisciplinary health residency. Method: qualitative study, carried out with multiprofessional residency programs in the state of Ceará and guided by the Competences in Health Promotion Project (CompHP) framework. Thirteen professionals from the faculty of five residency programs with priority performance in primary care participated. Data were collected through semi-structured interviews and interpreted using a dendrogram organized in ALCESTE. Results: two classes were originated relating the conceptions of the residency faculty collegiate about competences, competence in health promotion and its development from the training process of the multidisciplinary residency. The development of skills in health promotion in the multiprofessional residency involves the articulation of psychomotor, cognitive and affective attributes. Therefore, collective and multidisciplinary practices are valued, using communication strategies, popular education and identification of partnerships, among others. Final considerations and implications for practice:fostering the development of skills in health promotion contributes to overcoming traditional teaching models and health practices, however political, institutional and social aspects highlight the need for caution in the development of studies based on training of multidisciplinary teams, representing limitations of the study.

Keywords: Health Human Resource Training; Professional Competence; Competency-Based Education; Internship, nonmedical; Health Promotion

\section{RESUMEN}

Objetivo: reconocer la conformación de competencias en promoción de la salud en la residencia multidisciplinar de salud. Método: estudio cualitativo, realizado con programas de residencia multiprofesional en el estado de Ceará y guiado por el marco del Proyecto de Competencias en Promoción de la Salud (CompHP). Participaron trece profesionales de la facultad de cinco programas de residencia con actuación prioritaria en atención primaria. Los datos fueron recolectados a través de entrevistas semiestructuradas e interpretados mediante un dendrograma organizado en ALCESTE. Resultados: se originaron dos clases relacionando las concepciones del colegiado de la facultad de residencia sobre competencias, competencia en promoción de la salud y su desarrollo a partir del proceso formativo de la residencia multidisciplinar. El desarrollo de habilidades en promoción de la salud en la residencia multiprofesional implica la articulación de atributos psicomotores, cognitivos y afectivos. Por ello, se valoran las prácticas colectivas y multidisciplinares, utilizando estrategias de comunicación, educación popular e identificación de alianzas, entre otras. Consideraciones finales e implicaciones para la práctica: fomentar el desarrollo de habilidades en promoción de la salud contribuye a superar los modelos tradicionales de enseñanza y las prácticas de salud, sin embargo aspectos políticos, institucionales y sociales resaltan la necesidad de cautela en el desarrollo de estudios basados en la formación de equipos multidisciplinarios, representando limitaciones del estudio.

Palabras-clave: Capacitación de Recursos Humanos en Salud; Competencia Profesional; Educación Basada en Competencias; Internado no Médico; Promoción de la Salud. 


\section{INTRODUÇÃO}

A consolidação do Sistema Único de Saúde (SUS), ainda que diante das constantes ameaças ideológicas, políticas, econômicas e sociais retrógradas de manifestação hodierna na sociedade, alicerça-se na comprovação factual dos seus efeitos benéficos e resolutivos para com o bem-estar, qualidade de vida e promoção da saúde dos brasileiros.

Atuar efetivamente na promoção da saúde requer a articulação sinérgica de conhecimentos, habilidades e atitudes condizentes com a realidade e demandas de saúde da população. ${ }^{1}$ Para isso, o exercício profissional deve acontecer a partir de ações que envolvem, dentre outros requisitos, a autonomia, a ética, a capacidade de diagnosticar e solucionar problemas no âmbito da atenção e gestão do cuidado em saúde. ${ }^{2}$

Considerando a complexidade do alinhamento político e prático da assistência em saúde, ao longo dos últimos 30 anos, muitos debates têm sido levantados acerca das competências profissionais necessárias para tal. Essas discussões envolvem, portanto, os processos formativos e os lócus de aprendizagem em saúde. ${ }^{2}$

Nesse contexto, a residência multiprofissional em saúde se apresenta como um importante cenário, cuja dinâmica do processo formativo oferece significativo potencial para o desenvolvimento de competências em promoção da saúde junto aos atores sociais tais como residentes, preceptores, tutores e profissionais dos serviços de saúde articulados na sua composição. ${ }^{3}$

A residência multiprofissional fomenta a capacitação de recursos humanos em saúde pautada na educação permanente e educação baseada em competências, que busca substituir a lógica biologicista por um modo de pensar e conhecer capaz de apreender os objetos em seu contexto e sua complexidade, integrando saberes, apoiando-se na clínica ampliada, projeto terapêutico, trabalho interdisciplinar e multiprofissional. ${ }^{4}$

Esses aspectos coadunam com a proposta de requisitos profissionais para atuação em promoção da saúde do Competences in Health Promotion Project (CompHP). O CompHP reúne um conjunto de competências essenciais para a prática efetiva em promoção da saúde, formulado a partir de intercâmbios multivariados de conhecimentos de experts internacionais e ampla revisão da literatura, materializando-se como referência para as práticas, educação e formação para a promoção da saúde. ${ }^{1}$

O conjunto de competências essenciais, propostas pelo CompHP para desenvolver ações eficazes em promoção da saúde, abrange valores, conhecimento, habilidades e atitudes, listados em 47 competências agrupadas em nove domínios, a saber: 1) Favorecimento de mudanças; 2) Advocacia em saúde; 3) Parceria; 4) Comunicação; 5) Liderança; 6) Diagnóstico; 7) Planejamento; 8) Implementação; e 9) Avaliação e Pesquisa. ${ }^{5}$

É a partir da consolidação dessas competências profissionais que o residente, enquanto trabalhador do SUS, favorecerá a um profissional empenhado na transformação das práticas de saúde, promovendo inovações e proporcionando atenção e gestão qualificada e integrada. ${ }^{6}$ Assim sendo, questiona-se: Como as competências em promoção da saúde são compreendidas na formação da residência multiprofissional em saúde? Quais os recursos mobilizados para o seu desenvolvimento nesta formação?

Sob este prisma, objetivou-se reconhecer a conformação de competências em promoção da saúde na residência multiprofissional em saúde.

\section{MÉTODO}

Estudo exploratório, com abordagem qualitativa, realizado junto aos programas de Residência Multiprofissional em Saúde do estado do Ceará e ancorado no Competences in Health Promotion Project (CompHP), enquanto referencial teórico.

A escolha pelo Ceará, enquanto cenário macro deste estudo, é justificada pela sua contribuição na formação de profissionais de saúde e para o avanço das políticas públicas de saúde, contemplando um dos programas pioneiros na modalidade residência no contexto da saúde da família, bem como o programa com maior número de vagas por turma do país, com distribuição territorial em todas as macrorregiões de saúde estaduais.

Nesse contexto, e apreciando a priori os programas de residência multiprofissional com lócus de atuação essencial na atenção básica, considerou-se a totalidade de programas atuantes a pelo menos três meses, quais sejam: dois programas com instituição formadora no município de Sobral e três programas com instituição formadora no município de Fortaleza. Cabe destacar que durante o processo de execução deste estudo um novo programa de residências deu início a suas atividades no município de Crato, entretanto não compôs a amostra participante considerando seu caráter inicial de implementação.

A população de interesse foi constituída pelos profissionais engajados na condução pedagógica de programas de internato não médico do tipo residência multiprofissional em saúde, tais como coordenação geral, coordenação de ênfases e tutores, que foram convidados a participar do estudo, a partir de amostra intencional não probabilística, aqueles que atenderam aos critérios de elegibilidade: estar vinculado à instituição formadora responsável pela residência e atuar diretamente no colegiado docente, por no mínimo três meses, estimando-se ser este período suficiente para participação no planejamento e/ou execução das atividades pedagógicas tocantes à promoção da saúde e desenvolvimento de competências. Tratou-se como critério de exclusão: estar afastado de suas atividades, por quaisquer motivos, durante o período de coleta de dados.

$\mathrm{Na}$ Instituição A, 12 profissionais satisfaziam os critérios de inclusão e sete participaram do estudo. Na Instituição B, dos 11 profissionais elegíveis, seis participaram do estudo. Assim, delineou-se como amostra 13 profissionais.

Como técnica para coleta de dados foram realizadas entrevistas com os informantes-chave, guiadas por um roteiro semiestruturado que contemplou questões acerca das concepções de promoção da saúde no processo formativo e sua materialização nos momentos teóricos e/ou práticos.

$\mathrm{Na}$ aproximação com o cenário do estudo, fez-se o levantamento dos nomes e contatos dos informantes-chave. Em seguida, procedeu-se com o agendamento via telefônica 
das entrevistas, que aconteceram em momento, condição e local apropriado, conforme disponibilidade dos participantes. Quando da sua realização, após a leitura e assinatura do Termo de Consentimento, as falas foram registradas em áudio digital, para maior precisão das informações, perfazendo o tempo médio de 45 minutos cada.

Com vistas a garantir a fidedignidade das informações e uma maior consistência dos dados, quando da transcrição das falas no Microsoft Word 2013®, estas foram devolvidas aos participantes para leitura e confirmação e/ou complementaridade das ideias no tocante a sua fidedignidade narrativa, em atenção à técnica member check. Em seguida, esse material subsidiou a construção do corpus de análise, no formato Richtext, denominado CompRMS e processado pelo software Anályse Lexicale por Contexte d' um Esemble de Segments de Texte (ALCESTE) versão $2015 \circledR$.

Dentre as possibilidades interpretativas de dados sob a ótica da análise lexical, optou-se pela interpretação por meio da perspectiva do conteúdo, denominando-se e interpretando cada classe a partir das informações fornecidas pelo software enquanto categorias. Os resultados apresentados pelo ALCESTE se referem à representação das Unidades de Contexto Elementar (UCE), que consistem em fragmentos de texto com sentido completo, em classes, a partir da interrelação entre as palavras, as UCE e as próprias classes. Em seu processamento, são destacadas ainda as palavras plenas como representativas das UCE que compõem uma classe, sendo estas palavras que excedem os significados registrados em dicionários, uma vez que são influenciadas pelo contexto de inserção dos participantes, caracterizando seu campo de referência e discurso.

As classes originadas pelo ALCESTE são apresentadas por meio de um dendrograma descendente. Estas expressam as palavras que mais se aproximam pelo seu conteúdo simbólico e assim representam os núcleos do sentido das informações coletadas e subsidiaram a análise à luz do referencial adotado e da literatura pertinente.

Preocupou-se em atender aos aspectos éticos da pesquisa científica com seres humanos, estando o estudo autorizado após apreciação e aprovação pelo Comitê de Ética em Pesquisa da Universidade Regional do Cariri, segundo parecer de nº 1.973.784. Com vistas a garantir e assegurar o sigilo das informações, atribuíram-se codificações por meio da abreviação "Part" seguida do número de participação.

\section{RESULTADOS}

Participaram do estudo 13 profissionais, dos quais dois eram coordenadores e onze eram tutores. Em sua maioria, os participantes eram do sexo feminino (onze entrevistadas). A média da idade dos participantes foi de 36,8 anos, e tempo de atuação docente variou de oito meses a 11 anos, com média de três anos e seis meses nas atividades ligadas a residência multiprofissional.

A ocupação do cargo atual de tutoria ou coordenação perfaz um período mínimo de três meses e máximo de sete anos.
Destaca-se que para oito destes profissionais a experiência na docência dar-se na inserção na residência multiprofissional.

Quanto à formação destes profissionais, verificou-se variação entre os núcleos profissionais, marcados por presença de profissionais das ciências humanas e ciências da saúde, sendo: quatro enfermeiros, dois profissionais de educação física, dois assistentes sociais, dois psicólogos, um cirurgião-dentista, um fisioterapeuta e um historiador. No que se refere à pós-graduação destes profissionais, o maior título observado é o de mestre, correspondente a titulação de oito dos entrevistados, seguido pelo título de especialista, de cinco participantes.

O processamento das entrevistas no ALCESTE deu origem ao dendograma, e, considerando o princípio de independência entre ramificações distintas e íntima ligação entre as classes de uma mesma ramificação, bem como as formas/palavras plenas de cada segmento do corpus e UCE, classificou-se as classes em dois blocos, sendo de interesse o bloco intitulado Competências em promoção da saúde no contexto da residência multiprofissional em saúde, aqui apresentado conforme objetivo do estudo.

O bloco Competências em promoção da saúde no contexto da residência multiprofissional em saúde compreende duas classes, compostas por 86 UCE, que relacionam as concepções do colegiado docente da residência sobre competências, competência em promoção da saúde e seu desenvolvimento a partir do processo formativo da residência multiprofissional.

De modo ilustrativo, as classes em questão estão representadas no dendrograma (Figura 1), junto às formas reduzidas representativas da classe e a força de cada forma em meio a classe, figurada pelo qui-quadrado $(x 2)$.

\section{Competências em Promoção da Saúde: Conformações na Residência Multiprofissional em Saúde}

Esta categoria agrupa as conformações de competência em promoção da saúde no âmbito da formação da residência, fundamentando-se na concepção de competência enquanto uma articulação de conhecimentos, habilidades e atitudes para prática efetiva. Estas representam, neste âmbito, as dimensões: cognitiva (o saber), psicomotora (saber fazer) e afetiva (saber ser).

A análise lexical do ALCESTE demonstrou a prevalência do conhecimento $\left(\chi^{2}=80\right)$, seguida pela habilidade $\left(\chi^{2}=50\right)$, e por fim, pela atitude $\left(\chi^{2}=44\right)$. Esta sequência demonstra que há ainda a supervalorização do conhecimento sobre as habilidades e atitudes.

Os participantes do estudo apontaram o seguinte:

Uma competência para mim seria a união, a junção, entre conhecimentos teóricos, naturais, a respeito da temática, aliado as habilidades, quando o residente consegue justamente realizar essa construção daquilo que é preconizado pela literatura, digamos, aquela habilidade de lidar com determinada situação. (Par05) 


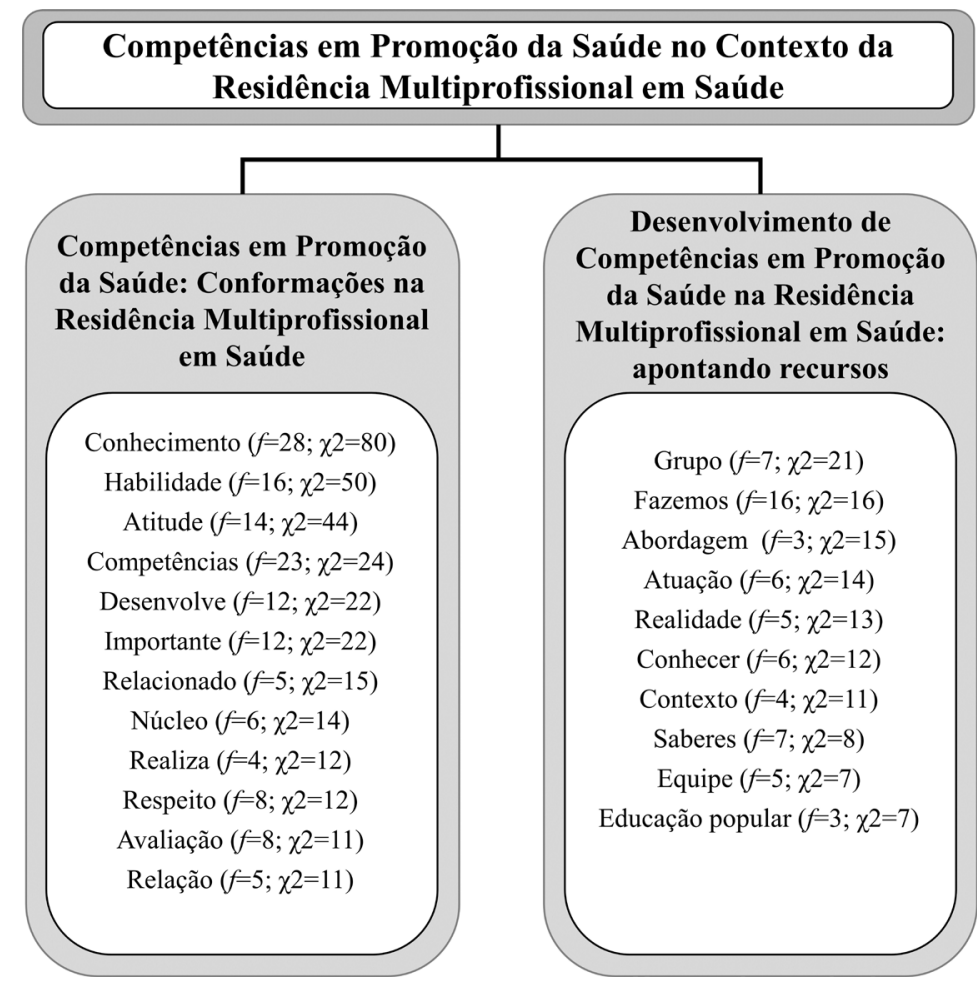

Figura 1. Dendograma de classificação ascendente do bloco Competências em promoção da saúde no contexto da residência multiprofissional em saúde. Crato, 2017.

Então as competências que a gente trabalha, dentro desse campo da residência, é você ter conhecimentos, habilidades e atitudes com relação a um determinado tema. (Par08)

Neste contexto, a concepção de atitude relaciona-se com as competências relacionais, como a amorosidade, que situam o ser em um meio social e exigem dele a adoção de posturas frente as diversas situações.

Percebemos que as competências relacionais, que agente chama transversais, como a amorosidade que é uma competência que colocamos, que é para se desenvolver durante os dois anos, por isso ela está em todos os ciclos, é transversal. Mas percebemos que ainda é uma coisa da pessoa, a pessoa ir compreendendo, ir transformando. (Par01)

As habilidades e atitudes ganham espaço no processo formativo da residência multiprofissional, visto, principalmente, a carga-horária destinada às práticas e vivências no território, que promovem o encontro com outros profissionais, indivíduos, famílias e comunidade, exercitando assim as interações interpessoais e o desenvolvimento de ações.

Neste caminhar, o progresso das competências na residência coincide com a superação de lacunas remanescentes da graduação dos profissionais de saúde e áreas afins pertencentes ao processo formativo, originadas, por vezes, devido a rápida condução das formações e predomínio de métodos tradicionais de ensino-aprendizagem.

A competência a partir do olhar educacional ela vem buscando e olhando a partir da construção de três pilares que são importantes, que é o conhecimento, as habilidades e as atitudes. Então, na residência é diferente de outras formações, de outros cursos, que muitas vezes foca muito na questão do conhecimento. (Par11)

Neste processo, emerge o desafio de avaliar o desempenho dos profissionais residentes frente a aquisição de conhecimentos, expansão e aperfeiçoamento de habilidade, e, principalmente, o incremento de atitudes, como demonstram as falas a seguir:

A gente tem mais segurança emavaliar as macrocompetências, mas as do núcleo (núcleo profissional de cada categoria) a gente ainda está engatinhando e tentando. (Par10)

Então a nossa dimensão da competência que a gente trabalha, ela vai se relacionar a cada ciclo, [...] essa parte dessa competência é uma parte que exige muito da gente para avaliar. (Par01)

Assim, desde sua concepção, os programas de residência multiprofissional em estudo preocupam-se com o desenvolvimento 
de competências e o direcionamento destas para a promoção da saúde.

\section{Desenvolvimento de Competências em Promoção da Saúde na Residência Multiprofissional em Saúde: apontando recursos}

Esta categoria reúne recursos apontados pelos profissionais participantes como essenciais para possibilitar o desenvolvimento de competências em promoção da saúde na residência multiprofissional, a saber: fortalecimento de grupos, educação popular, conhecimento do território, comunicação adequada, flexibilidade do trabalho e organização sistemática das ações de promoção da saúde.

O fortalecimento dos grupos diz respeito à valorização de práticas coletivas, principalmente por meio da implementação de grupos, quer seja criando, quer seja reforçando e aperfeiçoando os já existentes no território. Para tanto, deve-se ancorar nos saberes da educação popular, que considera os sujeitos como protagonistas de seu próprio processo de aprender e, deste modo, de produzir cuidado e saúde, como exemplificado pelos participantes:

Então existe a troca de saberes mesmo. Troca de saberes que a gente precisa ter com os profissionais, com a comunidade. (Par02)

Dentro da educação popular, que a gente trabalha bastante, cada uma vai tentando colaborar com outra, segundo o que já conhece ou vivenciou. Tentando fazer essa troca. E a gente fazendo só essa mediação, nesses grupos. (Par04)

A efetividade dos grupos está relacionada, ainda, ao uso de técnicas e tecnologias adequadas para a comunicação das ações de promoção da saúde, englobando a comunicação verbal, uso correto e contextualizado da linguagem e observação dinâmica do contexto através da comunicação não verbal.

Outra coisa que ele precisa ter como competência é a adequação daquilo que ele precisa trabalhar numa população, ou o nível de entendimento, a linguagem dos usuários, para justamente chegar lá no território e falar sobre pé diabético, num grupo de hipertensos e diabéticos e não utilizar uma abordagem meramente técnica. (Par05)

Pontua-se ainda a atuação a partir do reconhecimento das necessidades e ofertas do território, identificando atores-chave, dispositivos parceiros e estruturando um processo de trabalho voltado para a lógica territorial e práticas saudáveis.

Eu preciso conhecer para eu atuar. Eu preciso saber que contexto é esse que eu estou vivenciando. (Par02)
Vai fazer uma ação comunitária? Tu precisas conhecer, que território, que população, o que que é comum, como tu abordar. (Par06)

A atuação voltada para a promoção da saúde, por fim, exige o abandono de práticas prescritivas e engessadas em direção de um processo de trabalho dinâmico e flexível que se permita inventar e reinventar diante das possibilidades e necessidades de um território que é vivo e de uma população com demandas complexas, conforme exposto:

Eu preciso saber, conhecer e renovar o meu trabalho o tempo inteiro. Eu preciso rever, eu preciso avaliar e eu preciso modificar. (Par06)

\section{DISCUSSÃO}

No contexto em análise, reconhece-se uma supervalorização do conhecimento sobre as habilidades e atitudes. Essa concepção de competências, com ênfase no conhecimento, condizente com um processo histórico-cultural que permeia os processos educativos desde a Idade Média, é reflexo do caráter positivista, biologicista e médico - centrado que o processo formativo ainda sustenta.

A literatura mostra que no desenvolvimento de competências em promoção da saúde, não distante da educação tradicional, também é perceptível a dominância do conhecimento científico, ocupando posição hierárquica privilegiada às habilidades e atitudes, ao passo que já manifesta preocupação em transformar essa realidade. ${ }^{7}$

Ainda que haja uma maior valorização da dimensão conhecimento, algumas falas expressam que as competências se referem a uma articulação de aspectos que envolvem o saber, saber-fazer e ser. Essa lógica se aproxima com os pressupostos do CompHP acerca da definição de competências, estabelecendoas como requisitos essenciais para a atuação profissional. ${ }^{1,2}$

A conformação do processo formativo na residência coloca os profissionais no bojo da comunidade, para que, a partir da compreensão da realidade e do estabelecimento de vínculo com os usuários do serviço de saúde, os profissionais possam desenvolver ações que melhorem as condições de vida e saúde deles.

Vislumbra-se, portanto, um processo cíclico, bidimensional e de colaborações mútuas, onde as contribuições profissionais para o cuidado do indivíduo, da família e/ou da comunidade, reforçam e aperfeiçoam o próprio desenvolvimento desta prática. ${ }^{3}$ Sendo assim, o objeto de cuidado dos profissionais de saúde é, também, o eixo transformador da sua prática profissional.

O desenvolvimento de competências em promoção da saúde, especialmente no âmbito da atenção básica, exige a mobilização efetiva de processos atitudinais e psicomotores, posto que a complexidade vivenciada neste cenário requer que os profissionais apresentem mais que conhecimentos, 
contemplando valores éticos e responsabilidade social diante da comunidade, bem como o desenvolvimento de habilidades e atitudes. ${ }^{8}$

O desenvolvimento, aperfeiçoamento e fortalecimento dessas competências exige a exposição dos residentes a situações singulares nas quais se possa agir tecnologicamente com a realidade social e dialogar com esta, sendo a aproximação com a realidade dos serviços a oportunidade ideal para atingir esse objeto relacional. Isso reafirma que o progresso nas competências coincide com a superação de lacunas deixadas por formações no modelo tradicional. ${ }^{9}$

As porosidades percebidas na formação profissional a nível de graduação, mesmo frente aos avanços nas discussões no campo da educação, devem-se, em partes, à liberdade que as Instituições de Ensino Superior gozam em organizar seus cursos, os quais, respaldados pela Lei de Diretrizes e Bases da Educação Nacional (LDB), organizam os processos formativos para a preparação, quase que exclusivamente, para o mercado de trabalho, perpetuando um modelo de formação flexeneriano, biomédico e curativo. ${ }^{10}$

As atuais políticas e pressupostos da educação permanente em saúde sinalizam que a formação desses profissionais deve estimular a descoberta de novas e diversas formas de agir em promoção da saúde, direcionadas pelos princípios do SUS, com vistas a produzir conhecimentos relevantes para a prestação de cuidados, integrar o ensino e a produção de saúde, e estabelecer cooperação entre saúde e educação para qualificar a gestão, e fixar os profissionais nas redes do sistema de saúde público. ${ }^{10}$

Entretanto, reconhece-se os avanços já proporcionados pela implementação destas diretrizes que defendem a formação de profissionais preocupados com o coletivo, engajados em desenvolver ações de promoção, prevenção, recuperação e reabilitação, na perspectiva da integralidade da assistência, além do desenho e execução de currículos flexíveis dirigidos para a aquisição de competências profissionais através do uso de metodologias modernas de aprendizagem aplicadas em múltiplos cenários de ensino. ${ }^{10,11}$

Dentre as mudanças já perceptíveis está a promoção de "processos de aprender a ser, a viver junto e conhecer, formando profissionais com autonomia e capacidade de assegurar uma atenção à saúde integral e de qualidade". 11:234 Reconhecer e valorizar o desenvolvimento de competências na residência multiprofissional se mostra como alicerce para formação em promoção da saúde pois corrobora com a expansão de normas profissionais e sistemas de garantia de qualidade que confirmam a promoção da saúde como campo especializado de práticas. ${ }^{12}$

A abordagem baseada em competências compreende de modo lógico e sequencial a especificação dos problemas a serem abordados, sendo preferível aqueles que emergem do território e da prática profissional; identificação das competências necessárias; adaptação do currículo para atingir as competências; e avaliação das realizações e reconhecimento das lacunas. ${ }^{2}$

Quanto à avaliação destas competências, delineada como um desafio pelos participantes, é necessário pensar instrumentos que agrupem os conhecimentos, habilidades e atitudes que compõem as competências desejadas e como elas se manifestam nas atividades diárias dos processos formativos, para que um acompanhamento objetivo possa ser realizado.

Nessa perspectiva, o CompHP, a medida em que descreve as competências específicas para cada domínio, tanto contribu para o direcionamento do processo formativo, quanto permite um melhor acompanhamento do desenvolvimento destes. ${ }^{13}$

Diversas são as estratégias existentes para acompanhar o desenvolvimento de competências. Uma dessas estratégias, adequada para averiguar se uma proposta educativa conseguiu promover o desenvolvimento do perfil de competências proposto, consiste em comparar as percepções iniciais dos profissionais participantes da formação com os resultados ao final do curso. ${ }^{14}$ Neste caso, não se trata de uma avaliação de desempenho, mas da percepção dos residentes sobre seu domínio em relação às capacidades que subjazem os desempenhos.

$\mathrm{Na}$ realidade em estudo, algo semelhante já acontece no processo de avaliação dos residentes, que, por meio de uma avaliação dialógica ao longo do processo formativo, considera o consenso de percepções do residente avaliado e de seus preceptores, bem como de profissionais do serviço.

A proposta avaliativa fundamenta-se, então, em contemplar diferentes perspectivas e olhares sobre a adequação profissional, rompendo as barreiras da centralização do cuidado, fomentando assim, a articulação de saberes e práticas numa perspectiva inter e transdisciplinar.

No que concerne aos recursos mobilizados para desenvolvimento destas competências, as atividades grupais implementadas no contexto dos serviços de saúde se manifestaram como uma das principais estratégias desenvolvidas. Conforme expresso nas falas, estas apresentam conotação de educação em saúde, com base no modelo dialógico da educação popular.

Este modelo apresenta características comuns ao movimento da promoção da saúde ao ser pensada sobre a realidade dos indivíduos e do meio no qual estão inseridos, ao valorizar e incentivar as práticas coletivas. Sendo este um elemento chave para arregimentar mudanças na qualidade de vida e saúde dos sujeitos envolvidos.

Contemplando a educação popular, a educação em saúde exerce seu caráter educativo bilateralmente, onde ambas as partes envolvidas aprendem por meio de uma relação que promove a troca de saberes. Assim, as atividades em grupo caracterizam-se como um processo democrático e solidário. ${ }^{15}$

Sob este prisma, o uso correto e adequado da comunicação é fundamental para viabilizar uma assistência humanística voltada para as necessidades de saúde dos sujeitos, pois assegura a interação entre profissionais de saúde e usuários, famílias e comunidade, não só a partir de uma relação de cuidados, mas de sensibilidade, aceitação, empatia e troca de saberes. ${ }^{16}$

No território, especificamente o contexto de atuação da atenção básica, exige-se que os sujeitos se apropriem sobre estas manifestações, considerando as subjetividades, o imaginário 
e o potencial do local para reconhecer as necessidades, os atores-chave e as parcerias para o desenvolvimento de ações. ${ }^{17}$

Os territórios de atuação apresentam então, além de características físicas, como a delimitação espacial, compreendendo toda a construção social que nele se deu e que a ele deu origem, aspectos históricos, demográficos, epidemiológicos, administrativos, tecnológicos, políticos, sociais, culturais e ambientais que o torna vivo, em constante construção. ${ }^{17}$

Para uma efetiva atuação no âmbito da promoção da saúde, acredita-se ser necessário que a formação sensibilize o profissional para a criticidade dos aspectos que movimentam o fazer saúde, refletindo constantemente sobre as ações desenvolvidas e como estas podem ser aperfeiçoadas e estarem adequadas às realidades.

A dinâmica do processo de trabalho em promoção da saúde, capaz de transformar e modificar positivamente as condições de vida e saúde, só se viabiliza mediante profissionais críticos e com capacidade de flexibilizar-se aos contextos diversos com a organização sistemática de ações para o abandono de práticas prescritivas para ir de encontro ao modelo dinâmico, flexível e reinventado. ${ }^{10}$

Ressente-se que o processo formativo vivenciado na residência multiprofissional em saúde tem aspectos que coadunam com as propostas orientadoras definidas pelo CompHP, ao passo em que evidencia significativas aproximações teóricas e práticas com este referencial, indicando que os caminhos trilhados buscam direcionar-se para a formação de promotores de saúde, com base no desenvolvimento de competências essenciais, dentre elas comunicação, diagnóstico das demandas e realidades vividas, implementação e avaliação contínua dessas ações. ${ }^{1}$

\section{CONSIDERAÇÕES FINAIS E IMPLICAÇÕES PARA A PRÁTICA}

Considerando o exposto, tem-se que as competências em promoção da saúde na residência multiprofissional são compreendidas como a articulação sinérgica de atributos psicomotores, cognitivos e afetivos que contribuem com a superação de modelos tradicionais de ensino e práticas em saúde. Neste contexto, as habilidades e atitudes são reconhecidas e valorizadas na associação mútua com os conhecimentos, ainda identificado como dimensão centralizadora e ordenadora do processo ensino-aprendizagem

Em relação aos recursos mobilizados para tal, observa-se que no componente comunitário da residência multiprofissional existe a valorização de práticas coletivas, reafirmando o protagonismo dos envolvidos nos processos formativos implementados na atenção básica, uma vez que é oportuna a troca de saberes, utilização de estratégias de comunicação adequadas, a educação popular, o reconhecimento de necessidades do território e a identificação de parceiras para ações voltadas ao dinamismo, flexibilidade e reinvenção de práticas em saúde.

No entanto, reconhece-se que há diversos fatores, dentre eles políticos, institucionais e sociais, que destacam a necessidade de cautela no desenvolvimento de estudos cuja essência pauta-se na formação de equipes multiprofissionais, as quais podem ser delineadas a partir das realidades e demandas loco-regionais.

É mister ressaltar que, a medida em que esta premissa se manifesta como uma limitação deste estudo, sinaliza possibilidades para o desenvolvimento de pesquisas que possam investigar em profundidade os desafios aqui apontados. Aponta-se ainda que, embora os pesquisadores tenham realizado a validação narrativa junto aos informantes-chave de suas falas transcritas, a impossibilidade de avaliação interpretativa pode também ser pontuada como uma limitação deste estudo.

Destarte, delineiam-se aqui elementos essenciais ao desenvolvimento de competências em promoção da saúde na residência multiprofissional em saúde, potencializando o processo pedagógico e planejamento dos momentos formativos concernentes com o avanço das práticas em promoção da saúde, destacando a necessidade de considerar as particularidades do território e seus atores e partindo de um modelo dialógico e participativo de ensinar e aprender.

\section{FINANCIAMENTO}

O presente trabalho foi realizado com apoio da Fundação Cearense de Apoio ao Desenvolvimento Científico e Tecnológico (FUNCAP), por meio de bolsa de mestrado concedida ao desenvolvimento da dissertação "Competências em promoção da saúde no processo formativo da residência multiprofissional: um olhar sob a perspectiva do CompHP", de Lucas Dias Soares Machado.

\section{CONTRIBUIÇÕES DOS AUTORES}

Desenho do estudo: Lucas Dias Soares Machado. Maria de Fátima Antero Sousa Machado

Aquisição de dados: Lucas Dias Soares Machado.

Análise de dados e interpretação dos resultados: Lucas Dias Soares Machado. Samyra Paula Lustoza Xavier. Paloma Loiola Leite. Maria Rosilene Cândido Moreira. Maria Rocineide Ferreira Silva. Maria de Fátima Antero Sousa Machado.

Redação e revisão crítica do manuscrito: Lucas Dias Soares Machado. Samyra Paula Lustoza Xavier. Paloma Loiola Leite. Maria Rosilene Cândido Moreira. Maria Rocineide Ferreira Silva. Maria de Fátima Antero Sousa Machado.

Aprovação da versão final do artigo: Lucas Dias Soares Machado. Samyra Paula Lustoza Xavier. Paloma Loiola Leite. Maria Rosilene Cândido Moreira. Maria Rocineide Ferreira Silva Maria de Fátima Antero Sousa Machado.

Responsabilidade por todos os aspectos do conteúdo e a integridade do artigo publicado: Lucas Dias Soares Machado. Samyra Paula Lustoza Xavier. Paloma Loiola Leite. Maria Rosilene Cândido Moreira. Maria Rocineide Ferreira Silva. Maria de Fátima Antero Sousa Machado.

\section{EDITOR ASSOCIADO}

Maria Catarina Motta (D) 


\section{EDITOR CIENTÍFICO}

Marcelle Miranda da Silva (D)

\section{REFERÊNCIAS}

1. Battel-Kirk B, Barry MM. Evaluating progress in the uptake and impact of Health Promotion competencies in Europe. Health Promot Int. 2020;35(4):779-89. http://dx.doi.org/10.1093/heapro/daz068. PMid:31361299.

2. Xavier SPL, Pereira AP, Moreira MRC, Martins AKL, Ferreira HS, Machado MFAS. Competências em promoção a saúde à luz do projeto Competencies Health Promotion (CompHP): uma revisão integrativa. Cienc Cuid Saude. 2019;18(1)

3. Machado MFAS, Machado LDS, Xavier SPL, Lima LA, Moreira MRC, Ferreira HS. Competências em promoção da saúde: o domínio parceria na residência multiprofissional em saúde. Rev Bras Promoç Saúde. 2018;31(4):1-7. http://dx.doi.org/10.5020/18061230.2018.8761.

4. Bernardo MS, Fabrizzio GC, Souza ML, Santos TO, Andrade SR Training and work process in Multiprofessional Residency in Health as innovative strategy. Rev Bras Enferm. 2020;73(6):e20190635. http:// dx.doi.org/10.1590/0034-7167-2019-0635. PMid:32901748.

5. Battel-Kirk B, Barry MM. Has the development of health promotion competencies made a difference? A scoping review of the literature. Health Educ Behav. 2019;46(5):824-42. http://dx.doi.org/10.1177/1090198119846935. PMid:31064229.

6. Silva CT, Terra MG, Kruse MHL, Camponogara S, Xavier MS. Residência multiprofissional como espaço intercessor para a educação permanente em saúde. Texto Contexto Enferm. 2016;25(1):e2760014. http://dx.doi. org/10.1590/0104-0707201600002760014.

7. Netto L, Silva KL, Rua MS. Desenvolvimento de competências para promoção da saúde e mudança no modelo assistencial. Texto Contexto Enferm. 2016;25(2):e2150015.

8. Santana KFS, Machado LDS, Machado MFAS, Dias MAS, Silva LMS, Lopes MSV. Competences in health promotion in the environmental education practices of community health agents. Rev Gaúcha Enferm. 2021;42:e20200053. http://dx.doi.org/10.1590/1983-1447.2021.20200053. PMid:33566950.

9. Teófilo TJS, Santos NLP, Baduy RS. Betting on change in medical education: trajectories of a medical school. Interface. 2016;21(60):177-88.

10. Tavares MFL, Rocha RM, Bittar CML, Petersen CB, Andrade M. A promoção da saúde no ensino profissional: desafios na saúde e a necessidade de alcançar outros setores. Cienc Cuid Saude. 2016;21(6):1799-1808. http://dx.doi.org/10.1590/1413-81232015216.07622016.

11. Xavier SPL, Machado LDS, Moreira MRC, Martins AKL, Machado MFAS. Professional competencies to promote health in nursing and physical education undergraduate courses. Rev Bras Enferm. 2021;74(2):e20200617. http://dx.doi.org/10.1590/0034-7167-20200617. PMid:34076222.

12. Cestari VRF, Florêncio RS, Moreira TMM, Pessoa VLMP, Barbosa IV, Lima FET et al. Competências do enfermeiro na promoção da saúde de indivíduos com cardiopatias crônicas. Rev Bras Enferm. 2016;69(6):1195203. http://dx.doi.org/10.1590/0034-7167-2016-0312. PMid:27925098

13. Kane M, Royer-Barnett J, Alongi J. Core competencies for chronic disease prevention practice. Prev Chronic Dis. 2019;16:E144. http:// dx.doi.org/10.5888/pcd16.190101. PMid:31651380.

14. Gomes R, Padilha RQ, Lima VV, Silva CMFP. Avaliação de percepções sobre gestão da clínica em cursos orientados por competência. Cien Saude Colet. 2018;23(1):17-28. http://dx.doi.org/10.1590/141381232018231.26652015. PMid:29267808.

15. Silveira LMC, Ribeiro VMB. Grupo de adesão ao tratamento: espaço de "ensinagem" para profissionais de saúde e pacientes. Interface. 2005;9(16):91-104. http://dx.doi.org/10.1590/S1414-32832005000100008.

16. Netto L, Silva KL. Reflective practice and the development of competencies for health promotion in nurses' training. Rev Esc Enferm USP. 2018;52(0):e03383. http://dx.doi.org/10.1590/s1980220x2017034303383. PMid:30517299.

17. Calistro MO, Teixeira Y, Lacerda IRAS, Sousa SM, Agostinho Neto J, Duavy SMP et al. Territorialization using georreferencing and stratification of the social vulnerability of families in Primary Care. Cien Saude Colet. 2021;26(6):2141-8. http://dx.doi.org/10.1590/141381232021266.39402020. PMid:34231726. 